\title{
Chow rings of toric varieties defined by atomic lattices
}

\section{Journal Article}

\section{Author(s):}

Feichtner, Eva Maria; Yuzvinsky, Sergey

Publication date:

2004-03

Permanent link:

https://doi.org/10.3929/ethz-b-000051929

Rights / license:

In Copyright - Non-Commercial Use Permitted

\section{Originally published in:}

Inventiones mathematicae 155(3), https://doi.org/10.1007/s00222-003-0327-2 


\title{
Chow rings of toric varieties defined by atomic lattices
}

\author{
Eva Maria Feichtner ${ }^{1}$, Sergey Yuzvinsky $^{2}$ \\ 1 Department of Mathematics, ETH Zurich, 8092 Zurich, Switzerland \\ (e-mail: feichtne@math.ethz.ch) \\ 2 Department of Mathematics, University of Oregon, Eugene, OR 97403, USA \\ (e-mail: yuz@math. uoregon.edu)
}

Published online: 9 September 2003 - (C) Springer-Verlag 2003

\begin{abstract}
We study a graded algebra $D=D(\mathcal{L}, \mathscr{g})$ over $\mathbb{Z}$ defined by a finite lattice $\mathscr{L}$ and a subset $\mathscr{Q}$ in $\mathscr{L}$, a so-called building set. This algebra is a generalization of the cohomology algebras of hyperplane arrangement compactifications found in work of De Concini and Procesi [2]. Our main result is a representation of $D$, for an arbitrary atomic lattice $\mathcal{L}$, as the Chow ring of a smooth toric variety that we construct from $\mathcal{L}$ and $q$. We describe this variety both by its fan and geometrically by a series of blowups and orbit removal. Also we find a Gröbner basis of the relation ideal of $D$ and a monomial basis of $D$.
\end{abstract}

\section{Introduction}

In this article we study a graded algebra $D=D(\mathscr{L}, \mathscr{Q})$ over $\mathbb{Z}$ that is defined by a finite lattice $\mathcal{L}$ and a special subset, a so-called building set, $\mathscr{Q}$ in $\mathcal{L}$. The definition of this algebra is inspired by a presentation for the cohomology of arrangement compactifications as it appears in work of De Concini and Procesi [2].

In $[1,2]$ the authors studied a compactification of the complement of subspaces in a projective space defined by a building set in the intersection lattice $\mathcal{L}$ of the subspaces. In particular they gave a description of the cohomology algebra $H^{*}$ of this compactification in terms of generators and relations. In general, the set of defining relations for $H^{*}$ is much larger than the one we propose for $D$. However, in the case of all subspaces being of codimension 1 and $\mathcal{G}$ the set of irreducibles in $\mathcal{L}$, the former can be reduced to the latter [2, Prop. 1.1]. We show that this reduction holds for 
arbitrary building sets in $\mathcal{L}$, thus giving a first geometric interpretation of the algebra $D(\mathcal{L}, \mathscr{Q})$ (compare Corollary 2 ).

Our first result about $D$ is that for an arbitrary atomic lattice $\mathcal{L}$ a larger set of relations, similar to the defining relations of $H^{*}$, holds in $D$. To define the new relations for arbitrary lattices beyond the geometric context of arrangements, we need to introduce a special metric on the chains of $\mathcal{L}$. In fact, this new set of relations forms a Gröbner basis of the relation ideal which allows us to define a basis of $D$ over $\mathbb{Z}$ generalizing the basis defined in [9] and [7].

Our main result about $D$ motivating its definition is Theorem 3 which asserts that $D$ is naturally isomorphic to the Chow ring of a smooth toric variety $X=X_{\Sigma(\mathcal{L}, q)}$ constructed from an atomic lattice $\mathcal{L}$ and a building set $\mathcal{G}$ in $\mathcal{L}$. This result gives a second geometric interpretation of $D$, this time for arbitrary atomic lattices.

We introduce the toric variety $X$ by means of its polyhedral fan $\Sigma(\mathcal{L}, \mathscr{q})$ that we build directly from $\mathscr{L}$ and $\mathscr{g}$. Then we give a more geometric construction of $X$ as the result of several toric blowups of an affine complex space and subsequent removal of certain open torus orbits.

The article is organized as follows. In Sect. 2, we recall the necessary combinatorial definitions and define the algebra $D=D(\mathcal{L}, \mathcal{q})$. In Sect. 3 , we extend the set of relations for $D$ to a Gröbner basis of the relation ideal and exhibit a basis of the algebra. In Sect. 4, we review the De ConciniProcesi compactifications of arrangement complements and relate $D$ to their cohomology algebras. Also we give some examples of the Poincaré series of these compactifications using our basis. Section 5 is devoted to the definition of the toric variety $X$ from a pair $(\mathcal{L}, \mathcal{G})$. We prove our main theorem asserting that $D$ is naturally isomorphic to the Chow ring of $X$. In Sect. 6, we give another construction of $X$ as the result of a series of toric blowups and subsequent removal of some open orbits. Finally, in Sect. 7, we consider a number of simple examples.

\section{The algebra $D(\mathscr{L}, \mathscr{g})$}

We start with defining some lattice-theoretic notions, building sets and nested sets, that provide the combinatorial essence for our algebra definition below. These notions, in the special case of intersection lattices of subspace arrangements, are crucial for the arrangement model construction of De Concini and Procesi [1]. For our purpose, we choose to present purely order-theoretic generalizations of their notions that previously appeared in [4].

By a lattice, in this article, we mean a finite partially ordered set all of whose subsets have a least upper bound (join, $\vee$ ) and a greatest lower bound (meet, $\wedge$ ). The least element of any lattice is denoted by $\hat{0}$. For any subset $\mathcal{Q}$ of a lattice $\mathcal{L}$ we denote by $\max g$ the set of maximal elements 


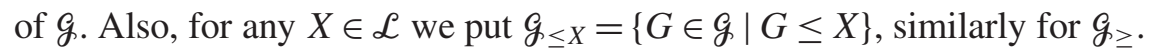
To denote intervals in $\mathcal{L}$ we use the notation $[X, Y]:=\{Z \in \mathcal{L} \mid X \leq Z \leq \bar{Y}\}$ for $X, Y \in \mathcal{L}$.

Definition 1. Let $\mathcal{L}$ be a finite lattice. A subset $\mathcal{G}$ in $\mathcal{L} \backslash\{\hat{0}\}$ is called $a$ building set in $\mathcal{L}$ if for any $X \in \mathcal{L} \backslash\{\hat{0}\}$ and $\max g_{\leq X}=\left\{G_{1}, \ldots, G_{k}\right\}$ there is an isomorphism of posets

$$
\varphi_{X}: \prod_{i=1}^{k}\left[\hat{0}, G_{i}\right] \stackrel{\cong}{\longrightarrow}[\hat{0}, X]
$$

with $\varphi_{X}\left(\hat{0}, \ldots, G_{i}, \ldots, \hat{0}\right)=G_{i}$ for $i=1, \ldots, k$. We call $\max g_{\leq X}$ the set of factors of $X$ in $g$.

As a first easy example one can take the maximal building set $\mathcal{L} \backslash\{\hat{0}\}$. Looking at the other extreme, the elements $X \in \mathcal{L} \backslash\{\hat{0}\}$ for which $[\hat{0}, X]$ does not decompose as a direct product, so-called irreducibles in $\mathcal{L}$, form the minimal building set in a given lattice $\mathcal{L}$.

The choice of a building set $\mathcal{G}$ in $\mathcal{L}$ gives rise to a family of nested sets. Roughly speaking these are the subsets of $\mathcal{L}$ whose antichains are sets of factors with respect to the building set $g$. The precise definition is as follows.

Definition 2. Let $\mathcal{L}$ be a finite lattice and $g$ a building set in $\mathcal{L}$. A subset 8 in $g$ is called nested if, for any set of pairwise incomparable elements $G_{1}, \ldots, G_{t}$ in 8 of cardinality at least two, the join $G_{1} \vee \cdots \vee G_{t}$ does not belong to $g$. The nested sets in $g$ form an abstract simplicial complex, the simplicial complex of nested sets $\mathcal{N}(\mathcal{L}, \mathscr{g})$.

For the maximal building set $\mathcal{G}=\mathcal{L} \backslash\{\hat{0}\}$ the nested set complex coincides with the order complex of $\mathcal{L} \backslash\{\hat{0}\}$. Smaller building sets yield nested set complexes with fewer vertices, but allow for more dense collections of simplexes.

An important property of a nested set is that for any two distinct maximal elements $X$ and $Y$ we have $X \wedge Y=\hat{0}$ (see [4, Prop. 2.5(1), 2.8(2)]).

We now have all notions at hand to define the main character of this article.

Definition 3. Let $\mathcal{L}$ be a finite lattice, $\mathfrak{A}(\mathcal{L})$ its set of atoms, and $\mathcal{G}$ a building set in $\mathcal{L}$. We define the algebra $D(\mathcal{L}, \mathscr{g})$ of $\mathcal{L}$ with respect to $\mathscr{g}$ as

$$
D(\mathcal{L}, g):=\mathbb{Z}\left[\left\{x_{G}\right\}_{G \in g}\right] / \tau
$$

where the ideal I of relations is generated by

$$
\prod_{i=1}^{t} x_{G_{i}} \quad \text { for }\left\{G_{1}, \ldots, G_{t}\right\} \notin \mathcal{N}(\mathcal{L}, \mathcal{g})
$$


and

$$
\sum_{G \geq H} x_{G} \quad \text { for } H \in \mathfrak{A}(\mathcal{L}) .
$$

Note that the algebra $D(\mathcal{L}, \mathscr{Q})$ is a quotient of the face algebra of the simplicial complex $\mathcal{N}(\mathcal{L}, \mathcal{Q})$. Although $D$ is defined for an arbitrary lattice our main constructions and results make sense only for atomic lattices, i.e., lattices in which any element is the join of some atoms. Thus we will restrict our considerations to this case.

In the special case of $\mathcal{L}$ being the intersection lattice of an arrangement of complex linear hyperplanes and $\mathscr{Q}$ being the minimal building set in $\mathcal{L}$, this algebra appears in work of De Concini and Procesi [2]. It is the cohomology algebra of a compactification of the projectivized arrangement complement; for details we refer to Sect. 4.

\section{Gröbner basis}

The set of generators of the ideal $\tau$ in Definition 3, while being elegant, is too small for being a Gröbner basis of this ideal. In this section, we extend this set to a Gröbner basis. In particular, we will obtain a $\mathbb{Z}$-basis of $D(\mathcal{L}, \mathcal{Q})$.

To define the larger set of relations we need to introduce a metric on chains in $\mathcal{L}$.

Definition 4. Let $\mathcal{L}$ be an atomic lattice and $X, Y \in \mathcal{L}$ with $X \leq Y$. We denote by $d(X, Y)$ the minimal number of atoms $H_{1}, \ldots, H_{d}$ in $\mathcal{L}$ such that $Y=X \vee \bigvee_{i=1}^{d} H_{i}$.

The following four properties of the function $d$ are immediate:

(i) $d(X, Z) \geq d(Y, Z)$ for $X, Y, Z \in \mathcal{L}$ with $X \leq Y \leq Z$. Notice that equality is possible even if all three $X, Y$, and $Z$ are distinct. Also it is not necessarily true that $d(X, Y) \leq d(X, Z)$.

(ii) $d(X, Y)+d(Y, Z) \geq d(X, Z)$ for $X, Y, Z \in \mathcal{L}$ with $X \leq Y \leq Z$.

(iii) $d(X \vee Z, Y \vee Z) \leq d(X, Y)$ for $X \leq Y \in \mathscr{L}$ and $Z \in \overline{\mathcal{L}}$ arbitrary.

(iv) $d(A, A \vee B) \leq d(A \wedge B, B)$ for $A, B \in \mathcal{L}$.

For example, (iv) follows from the fact that if $(A \wedge B) \vee \bigvee_{i} H_{i}=B$ for some atoms $H_{1}, \ldots, H_{d}$ then $A \vee \bigvee_{i} H_{i}=A \vee B$. If $\mathcal{L}$ is geometric (for instance, the intersection lattice of a hyperplane arrangement) then $d(X, Y)=\operatorname{rk} Y-\operatorname{rk} X$ whence in (ii) equality holds and (iv) is the semimodular inequality.

Now we can introduce the new set of generators for $\mathcal{L}$. The new relations are analogous to the defining relations for the cohomology algebra of the compactification of the complement of an arrangement of projective subspaces described in [1]. 
Theorem 1. The ideal of relations $\mathcal{I}$ in Definition 3 is generated by polynomials of the following type:

$$
\begin{aligned}
& h_{\S}=\prod_{G \in \S} x_{G} \quad \text { for } s \notin \mathcal{N}(\mathcal{L}, \mathcal{g}), \\
& g_{\mathcal{H}, B}=\prod_{i=1}^{k} x_{A_{i}}\left(\sum_{G \geq B} x_{G}\right)^{d},
\end{aligned}
$$

where $A_{1}, \ldots, A_{k}$ are maximal elements in a nested set $\mathcal{H} \in \mathcal{N}(\mathscr{L}, \mathcal{G})$, $B \in g$ with $B>A=\bigvee_{i=1}^{k} A_{i}$, and $d=d(A, B)$.

Proof. First notice that polynomials (1) and (2) are among polynomials $h_{\&}$ and $g_{\mathcal{H}, B}$. (To see that polynomials (2) are among $g_{\mathcal{H}, B}$ choose $\mathcal{H}=\emptyset$, and $B=H \in \mathfrak{A}(\mathcal{L})$. Here and everywhere we use the usual agreement that the join of the empty set is $\hat{0}$.) Hence it is left to show that any $g_{\mathcal{H}, B}$ is in $\mathcal{I}$, i.e., it is a combination of polynomials (1) and (2).

We prove our claim by induction on $d$.

$\underline{d=1}$. Choose an atom $H$ of $\mathcal{L}$ with $H \vee A=B$. Then using (2) we have

$$
\prod_{i=1}^{k} x_{A_{i}}\left(\sum_{G \geq H} x_{G}\right) \in \mathcal{L} .
$$

We want to show that for any $G \geq H,\left\{G, A_{1}, \ldots, A_{k}\right\} \in \mathcal{N}=\mathcal{N}(\mathscr{L}, \mathcal{g})$ implies that $G \geq B$. Then, any summand with $G \geq B$ can be omitted from (5) using polynomials (1), and we obtain $g_{\mathscr{H}, B} \in \mathcal{I}$ for $d=1$.

First note that $G$ cannot be smaller than or equal to any of the $A_{i}$, $i=1, \ldots, k$, since $G \leq A_{i}$ would imply $H \leq A_{i}$ contradicting the choice of $H$.

Assume that $G$ is incomparable with $A_{1}, \ldots, A_{s}$ for some $s \geq 1$, and $G \geq A_{i}$ for $i=s+1, \ldots, k$. Since $\left\{G, A_{1}, \ldots, A_{k}\right\} \in \mathcal{N}$ these elements are the factors of the $g$-decomposition in

$$
\widetilde{G}:=G \vee \bigvee_{i=1}^{s} A_{i}=G \vee \bigvee_{i=1}^{k} A_{i} \geq H \vee \bigvee_{i=1}^{k} A_{i}=B
$$

Since $B \in g$, the elements $A_{i}, i=1, \ldots, s$, are not maximal in $g$ below $\widetilde{G}$, which contradicts the $A_{i}$ being factors of $\widetilde{G}$.

We conclude that $G$ is comparable with, i.e., larger than all $A_{i}$ whence $G \geq \bigvee_{i=1}^{k} A_{i} \vee H=B$.

$d>1$. Choose an atom $H$ of $\mathcal{L}$ from the set of atoms in the definition of $\overline{d(A, B)}$. Then $A<A \vee H<B$. Using (2) we have

$$
\prod_{i=1}^{k} x_{A_{i}}\left(\sum_{G \geq H} x_{G}\right)\left(\sum_{G \geq B} x_{G}\right)^{d-1} \in \mathcal{I} .
$$


We show, using polynomials (1) and (2) and the induction hypothesis, that any $G$ with $G \not B$ can be omitted from the first sum modulo $\mathcal{I}$.

Let $G_{0} \in g, G_{0} \geq H$ but $G_{0} \geq B$. Using polynomials (1) we can assume that $\left\{G_{0}, A_{1}, \ldots, A_{k}\right\} \in \mathcal{N}$. Due to the choice of $H, G_{0}$ cannot be smaller than any of the $A_{i}$. Further note that if $G_{0}$ is incomparable with say $A_{1}, \ldots A_{s}, s \leq k$, then it is incomparable also with all $A_{1}, \ldots, A_{k}$. Indeed the join $G_{0} \vee A_{1} \vee \ldots \vee A_{s}=G_{0} \vee A_{1} \vee \ldots \vee A_{k}$ is a $g$-decomposition. Hence the two following cases remain to be considered.

Case 1. $G_{0}$ is comparable with all $A_{i}, i=1, \ldots, k$, hence $G_{0} \geq A$. Our goal is to rewrite

$$
x_{G_{0}}\left(\sum_{G \geq B} x_{G}\right)^{d-1}
$$

modulo $\mathcal{I}$ so that it contains an expression of the form (4) with exponent $<d$ as a factor. First observe that $G_{0} \vee B \in g$ since $G_{0}, B \in g$ but $H<G_{0} \wedge B$ [1, Thm. 2.3, 3b']. The building set element $G_{0} \vee B$ is to take the role of $B$ in (4).

Let $G \in g$ with $G \geq B$. We want to show that any $G$ with $G \geq G_{0} \vee B$ can be omitted from (7) modulo $\mathcal{L}$. We can assume that $\left\{G, G_{0}\right\} \in \mathcal{N}$. If $G \leq G_{0}$ then $B \leq G_{0}$, contradicting the choice of $G_{0}$. If $G$ and $G_{0}$ were incomparable then $G \vee G_{0} \notin g$ contradicting the fact that they both are greater than $H$. Hence $G \geq G_{0}$ and thus $G \geq G_{0} \vee B$.

Thus (7) reduces to

$$
x_{G_{0}}\left(\sum_{G \geq G_{0} \vee B} x_{G}\right)^{d-1} .
$$

Using properties (iv) and (i) of our metric $d$ we obtain

$$
d\left(G_{0}, G_{0} \vee B\right) \leq d\left(G_{0} \wedge B, B\right) \leq d(A \vee H, B)<d .
$$

Hence (8) contains a polynomial of the form (4) with exponent $<d$ as a factor whence it lies in $\mathcal{I}$ by the induction hypothesis.

Case 2. $G_{0}$ is incomparable with $A_{1}, \ldots, A_{k}$.

Since $\left\{G_{0}, A_{1}, \ldots A_{k}\right\} \in \mathcal{N}$ we have $\widetilde{G}_{0}:=G_{0} \vee A_{1} \vee \ldots \vee A_{k} \notin g$. We want to rewrite

$$
\left(\prod_{i=1}^{k} x_{A_{i}}\right) x_{G_{0}}\left(\sum_{G \geq B} x_{G}\right)^{d-1}
$$

modulo $\mathcal{I}$ so that it contains a polynomial of the form (4) with exponent $<d$ as a factor.

Observe that $\widetilde{G}_{0} \vee B=G_{0} \vee B$, and, as in Case $1, G_{0} \vee B \in G_{\text {. Th }}$ This time, $\widetilde{G}_{0} \vee B=G_{0} \vee B$ is to take the role of $B$, and $\widetilde{G}_{0}$ the role of $A$ in (4). 
As in Case 1, we see that

$$
\begin{aligned}
\left(\prod_{i=1}^{k} x_{A_{i}}\right) x_{G_{0}}\left(\sum_{G \geq B} x_{G}\right)^{d-1} \equiv \\
\left(\prod_{i=1}^{k} x_{A_{i}}\right) x_{G_{0}}\left(\sum_{G \geq G_{0} \vee B} x_{G}\right)^{d-1} \text { modulo } \mathcal{L},
\end{aligned}
$$

arguing as before for nested pairs $\left\{G, G_{0}\right\}$.

Now the right hand side has a factor of the form (4) with exponent $<d$ because again

$$
d\left(\widetilde{G}_{0}, \widetilde{G}_{0} \vee B\right) \leq d\left(\widetilde{G}_{0} \wedge B, B\right) \leq d(B, A \vee H)<d
$$

This implies that the right hand side lies in $\mathcal{I}$ by the induction hypothesis which completes the proof.

The main feature of the new generating set is that it is a Gröbner basis of $\tau$. As the main reference for Gröbner bases we use [3]. Fix a linear order on $\mathcal{G}$ that refines the reverse of the partial order on $\mathcal{L}$. It defines a lexicographic order on the monomials which we use in the following theorem.

Theorem 2. The generating system (3) and (4) is a Gröbner basis of I.

Proof. To prove that a set of monic polynomials is a Gröbner basis for the ideal it generates it suffices to consider all pairs of their initial monomials with a common indeterminate, compute their syzygies, and show that these syzygies have standard expressions in generators (without remainders). We will prove this by a straightforward calculation. To make the calculation easier to follow we will use several agreements. For any polynomial $p \in \mathcal{I}$ we will be dealing with, we will exhibit a generator $g$ whose initial monomial in $(g)$ divides a monomial $\mu$ of $p$ and call $p-c(\mu) \frac{\mu}{i n(g)} g$ the reduction of $p$ by $g$ (here $c(\mu)$ is the coefficient of $\mu$ in $p$ ). Reducing a polynomial all the way to 0 gives a standard expression for it. Also since reduction by monomial generators is very simple we will not name specific generators of the form $h_{\&}$ but just call this reduction $h$-equivalence.

We use certain new notation in the proof. For each $s \subset g$ put $\pi_{s}=\prod_{A \in \mathcal{S}} x_{A}$ and for any $B \in g_{\text {put }} y_{B}=\sum_{Y \in g_{>B}} x_{Y}$.

Now we consider pairs $\left(g_{1}, g_{2}\right)$ of generators of $\tau$ of several types.

1. At least one of the generators is $h_{\S}$. If they both are of this type then the syzygy is 0 . If the other one is $g_{\mathscr{H}, B}$ with $B \notin \delta$ then the syzygy is divisible by $h_{\&}$ whence $h$-equivalent to 0 . Finally if $B \in \&$ then the only nontrivial case is where $T=(\& \cup \mathscr{H}) \backslash\{B\} \in \mathcal{N}=\mathcal{N}(g, \mathcal{L})$. Notice that then $\& \cup \mathcal{H} \notin \mathcal{N}$. The syzygy is $h$-equivalent to $\pi_{T} y_{B}^{d(A, B)}$ where $A=\bigvee_{X \in \mathscr{H}} X$ as usual. Put $\bar{A}=\bigvee_{X \in T} X$. If $X \in g_{>B}$ and $X \leq \bar{A} \vee B$ then $X$ cannot form a nested set 
with $T$. Indeed, if it did then $\bar{A} \vee B=X \vee \bar{A} \notin \mathcal{G}$ contradicting $T \cup\{X\} \notin \mathcal{N}$. Similarly, if $X \in G_{>B}$ and $X$ is incomparable with $\bar{A}$ then $X$ cannot form a nested set with $T$. Indeed, if it did then $X \vee(\bar{A} \vee B)=X \vee \bar{A} \notin \mathcal{N}$ implying that $X$ forms a nested set with $\& \cup \mathcal{H}$. This would contradict $X>B$.

Now using property (i) of the metric $d$ we can reduce the syzygy to 0 by $g_{T, \bar{A} \vee B}$.

For the rest of the proof we need to consider only pairs with $g_{i}=g_{\mathscr{H}_{i}, B_{i}}$ $(i=1,2)$. We denote the exponent of $x_{B_{i}}+y_{B_{i}}$ in $g_{i}$ by $d_{i}$.

2. Suppose $B_{1} \neq B_{2}$ and $B_{i} \notin \mathscr{H}_{j}$. In this case the syzygy is

$$
\pi_{\mathscr{H}_{2} \backslash \mathscr{H}_{1}} g_{1}\left(g_{1}-i n\left(g_{1}\right)\right)-\pi_{\mathscr{H}_{1} \backslash \mathscr{H}_{2}} g_{2}\left(g_{2}-i n\left(g_{2}\right)\right)
$$

and this is in fact a standard expression for it. (Here and to the end of the proof we use $\pi_{\S}$ for arbitrary subsets $\delta$ of $\mathcal{L}$ meaning that if $\delta$ is not nested the product is $h$-equivalent to 0 .)

3. Suppose $B_{1}=B_{2}=B$ and $d=d_{2}-d_{2} \geq 0$. Then the syzygy is

$$
\pi_{\mathscr{H}_{1} \cup \mathscr{H}_{2}}\left[x_{B}^{d}\left(x_{B}+y_{B}\right)^{d_{1}}-\left(x_{B}+y_{B}\right)^{d_{2}}\right]
$$

and it reduces to 0 by $g_{1}$.

4. At last, suppose $B_{1} \in \mathscr{H}_{2}$. Put $\mathscr{H}=\left(\mathscr{H}_{1} \cup \mathscr{H}_{2}\right) \backslash\left\{B_{1}\right\}$ and $x_{B_{i}}=x_{i}$, $y_{B_{i}}=y_{i}$. Then the syzygy is

$$
s=\pi_{\mathscr{H}}\left[\left(x_{1}+y_{1}\right)^{d_{1}} x_{2}^{d_{2}}-x_{1}^{d_{1}}\left(x_{2}+y_{2}\right)^{d_{2}}\right] .
$$

Adding to $s$ the polynomial $f=\pi_{\mathcal{H}}\left(x_{1}+y_{1}\right)^{d_{1}}\left[\left(x_{2}+y_{2}\right)^{d_{2}}-x_{2}^{d_{2}}\right]$ we obtain

$$
s^{\prime}=s+f=\pi_{\mathscr{H}}\left[\left(x_{1}+y_{1}\right)^{d_{1}}-x_{1}^{d_{1}}\right]\left(x_{2}+y_{2}\right)^{d_{2}} .
$$

Notice that $f$ is divisible by $g_{1}$ and $\operatorname{in}(f) \leq i n(s)$. Thus it suffices to reduce $s^{\prime}$ to 0 . Also we can immediately reduce $s^{\prime}$ by $g_{2}$ to

$$
s^{\prime \prime}=\pi_{\mathscr{H}} y_{1}^{d_{1}}\left(x_{2}+y_{2}\right)^{d_{2}} .
$$

For the next steps we sort out summands of $y_{1}$. Using property (i) of the metric $d$ we can delete the summands $x_{Y}$ with $B_{1}<Y<B_{2}$ reducing by $g_{\mathscr{H} \cup\{Y\}, B_{2}}$. The sum of all summands $x_{Y}$ with $Y \geq B_{2}$ forms $\pi_{\mathcal{H}}\left(x_{2}+y_{2}\right)^{d_{1}+d_{2}}$ that reduces to 0 by $g_{\mathscr{H}, B_{2}}$. Indeed, denote the join of $\mathscr{H}_{i}$ by $C_{i}$ and the join of $\mathscr{H}_{2} \backslash\left\{B_{1}\right\}$ by $C_{2}^{\prime}$. This gives the join of $\mathscr{H}_{\text {as }} C_{1} \vee C_{2}^{\prime}$. Then, using properties (ii) and (iii) of the metric $d$, we have

$$
\begin{aligned}
d\left(C_{1} \vee C_{2}^{\prime}, B_{2}\right) & \leq d\left(C_{1} \vee C_{2}^{\prime}, B_{1} \vee C_{2}^{\prime}\right)+d\left(B_{1} \vee C_{2}^{\prime}, B_{2}\right) \\
& \leq d\left(C_{1}, B_{1}\right)+d\left(C_{2}, B_{2}\right)=d_{1}+d_{2}
\end{aligned}
$$

After the reductions in the previous paragraph we are left with a sum each summand of which is divisible by a polynomial

$$
t_{Z}=\pi_{\mathscr{H}} x_{Z}\left(x_{2}+y_{2}\right)^{d_{2}},
$$


where $Z \in \mathscr{G}_{>B_{1}}, Z$ is incomparable with $B_{2}$, and $\mathscr{H} \cup\{Z\} \in \mathcal{N}$. To reduce this polynomial we sort out the summands in the second sum. If $Y \in G_{\geq} B_{2}$ is not greater than or equal to $Z \vee B_{2}$ then it is incomparable with $Z$ whence $\{Z, Y\} \notin \mathcal{N}$ since $B_{1}<Z, Y$. This implies that $t_{Z}$ is $h$-equivalent to

$$
t_{Z}^{\prime}=\pi_{\mathcal{H}} x_{Z}\left(\sum_{Y \geq B_{2} \vee Z} x_{Y}\right)^{d_{2}} .
$$

Finally $t_{Z}^{\prime}$ reduces to 0 by $g_{\mathcal{H} \cup\{Z\}, B_{2} \vee Z}$ since, by property (iii) of the metric $d$, we have

$$
d\left(C_{2}^{\prime} \vee Z, B_{2} \vee Z\right)=d\left(C_{2} \vee Z, B_{2} \vee Z\right) \leq d\left(C_{2}, B_{2}\right)=d_{2}
$$

This reduction completes the proof.

Corollary 1. The following monomials form a $\mathbb{Z}$-basis of the algebra $D(\mathcal{L}, \mathscr{Q})$ :

$$
\prod_{A \in S} x_{A}^{m(A)},
$$

where $\&$ is running over all nested subsets of $g$ and $m(A)<d\left(A^{\prime}, A\right)$, $A^{\prime}$ being the join of $\& \cap \mathcal{L}_{<A}$.

If $\mathcal{L}$ is the intersection lattice of a complex central hyperplane arrangement then this basis coincides with the basis exhibited in [9]. In the next section we will give some examples of computing the Hilbert series of the algebra using this basis.

\section{Arrangement compactifications}

As we mentioned before, for a geometric lattice the metric $d$ defined in Sect. 3 coincides with the difference of ranks. This holds in particular for the intersection lattice of a hyperplane arrangement. In this setting and for $g$ being the minimal building set, the algebra $D(\mathcal{L}, \mathscr{g})$ appeared in [2] as the cohomology algebra of a compactification of the projectivized arrangement complement. From our work in previous sections we can conclude that for any building set $g$ in $\mathcal{L}$ the algebra $D(\mathcal{L}, \mathscr{g})$ can be interpreted geometrically as the cohomology algebra of the corresponding arrangement compactification.

We first review the construction of arrangement models due to De Concini and Procesi in the special case of complex hyperplane arrangements [1].

Let $\mathcal{A}=\left\{H_{1}, \ldots, H_{n}\right\}$ be an arrangement of complex linear hyperplanes in $\mathbb{C}^{d}$. Factoring by $\bigcap H_{i}$ if needed, we can assume $\mathcal{A}$ to be essential, i.e., $\bigcap H_{i}=\{0\}$. The combinatorial data of such an arrangement is customarily recorded by its intersection lattice $\mathcal{L}(\mathcal{A})$, i.e., the poset of intersections of all subsets of hyperplanes ordered by reverse inclusion. The greatest element of $\mathcal{L}(\mathcal{A})$ is 0 and the least element is $\mathbb{C}^{d}$. Let $g \subseteq \mathcal{L}(\mathcal{A})$ be a building set in $\mathscr{L}(\mathcal{A})$, and let us assume here that $0 \in \mathcal{G}$. 
We define a map on $\mathcal{M}(\mathcal{A}):=\mathbb{C}^{d} \backslash \bigcup \mathcal{A}$, the complement of the arrangement,

$$
\Phi: \quad \mathcal{M}(\mathcal{A}) \longrightarrow \mathbb{C}^{d} \times \prod_{G \in \mathscr{G}} \mathbb{P}\left(\mathbb{C}^{d} / G\right)
$$

where $\Phi$ is the natural inclusion into the first factor and the natural projection to the other factors restricted to $\mathcal{M}(\mathcal{A})$. The map $\Phi$ defines an embedding of $\mathcal{M}(\mathcal{A})$ in the right hand side space and we let $Y_{g}$ denote the closure of its image. The space $Y_{g}$ is a smooth algebraic variety containing $\mathcal{M}(\mathcal{A})$ as an open set. The complement $Y_{g} \backslash \mathcal{M}(\mathcal{A})$ is a divisor with normal crossings with irreducible components indexed by building set elements. An intersection of several components is non-empty (moreover, transversal and irreducible) if and only if the index set is nested as a subset of $q$ $[1,3.1,3.2]$.

There is a projective analogue of $Y_{g}$. Consider the projectivization $\mathbb{P} \mathcal{A}$ of $\mathcal{A}$, i.e., the family of codim 1 projective spaces $\mathbb{P} H$ in $\mathbb{C P}^{d-1}$ for $H \in \mathcal{A}$. The following construction yields a compactification of the complement $\mathcal{M}(\mathbb{P} \mathcal{A}):=\mathbb{C P}^{d-1} \backslash \bigcup \mathbb{P} \mathcal{A}$. The map $\Phi$ described above is $\mathbb{C}^{*}$-equivariant, where $\mathbb{C}^{*}$ acts by scalar multiplication on $\mathcal{M}(\mathcal{A})$ and on $\mathbb{C}^{d}$, and trivially on $\prod_{G \in G} \mathbb{P}\left(\mathbb{C}^{d} / G\right)$. We obtain a map

$$
\bar{\Phi}: \quad \mathcal{M}(\mathbb{P} \mathcal{A}) \longrightarrow \mathbb{C P}^{d-1} \times \prod_{G \in \mathscr{q}} \mathbb{P}\left(\mathbb{C}^{d} / G\right),
$$

and again take the closure of its image to define a model $\overline{Y_{g}}$ for $\mathcal{M}(\mathbb{P} \mathcal{A})$. The space $\overline{Y_{g}}$ is a smooth projective variety and the complement $\overline{Y_{g}} \backslash \mathcal{M}(\mathbb{P} \mathcal{A})$ is a divisor with normal crossings. Irreducible components are indexed by building set elements in $g^{0}:=g \backslash\{\{0\}\}$, and intersections of irreducible components are non-empty if and only if corresponding index sets are nested in $g$.

Geometrically, the arrangement models $Y_{g}$ and $\overline{Y_{g}}$ are related as follows. The model $Y_{g}$ is the total space of a line bundle over $\overline{Y_{g}}$; in fact, it is the pullback of the tautological bundle on $\mathbb{C P}^{d-1}$ along the canonical map $\overline{Y_{g}} \rightarrow \mathbb{C P}^{d-1}$. In particular, $\overline{Y_{g}}$ is isomorphic to the divisor in $Y_{g}$ associated to $0[1,4.1]$.

Example 1. Let $\mathcal{A}_{n-1}$ denote the rank $n-1$ complex braid arrangement, i.e., the family of partial diagonals, $H_{i, j}: z_{j}-z_{i}=0,1 \leq i<j \leq n$, in $\mathbb{C}^{n}$. Its intersection lattice $\mathcal{L}\left(\mathcal{A}_{n-1}\right)$ equals the lattice $\Pi_{n}$ consisting of the set partitions of $[n]:=\{1, \ldots, n\}$ ordered by reverse refinement. The set $\mathcal{F}$ of partitions with exactly one block of size $\geq 2$ forms the minimal building set in $\Pi_{n}$. The De Concini-Procesi arrangement compactification $\overline{Y_{\mathcal{F}}}$ is isomorphic to the Deligne-Knudson-Mumford compactification of the moduli space $M_{0, n+1}$ of $n+1$-punctured complex projective lines [1, 4.3]. 
In the more general setting of affine models for complex subspace arrangements, De Concini and Procesi provide explicit presentations for the cohomology algebras of irreducible components of divisors and of their intersections in terms of generators and relations [1, §5]. As mentioned above, the compactification of a complex hyperplane arrangement $\overline{Y_{g}}$ is isomorphic to the divisor associated with the maximal building set element in the corresponding affine model. We recall a description of its integral cohomology algebra.

Proposition 1. ([1, Thm. 5.2]) Let $\mathcal{A}$ be an essential arrangement of complex hyperplanes, $\mathcal{L}=\mathcal{L}(\mathcal{A})$ its intersection lattice, and $\mathcal{g}$ a building set in $\mathcal{L}$ containing $\{0\}$. Then the integral cohomology algebra of the arrangement compactification $\overline{Y_{g}}$ can be described as

$$
H^{*}\left(\overline{Y_{g}}\right) \cong \mathbb{Z}\left[\left\{c_{G}\right\}_{G \in g}\right] / \mathcal{g},
$$

with generators $c_{G}, G \in G$, corresponding to the cohomology classes of irreducible components of the normal crossing divisor, thus having degree 2.

The ideal of relations $\mathrm{g}$ is generated by polynomials of the following type:

$$
\begin{aligned}
& \prod_{i=1}^{t} c_{G_{i}} \quad \text { for }\left\{G_{1}, \ldots, G_{t}\right\} \notin \mathcal{N}(\mathcal{L}, \mathcal{g}), \\
& \prod_{i=1}^{k} c_{A_{i}}\left(\sum_{G \geq B} c_{G}\right)^{d},
\end{aligned}
$$

where $A_{1}, \ldots, A_{k}$ are maximal elements in a nested set $\mathcal{H} \in \mathcal{N}(\mathcal{L}, \mathcal{g})$, $B \in g$ with $B>\bigvee_{i=1}^{k} A_{i}$, and $d=\operatorname{codim}_{\mathbb{C}} B-\operatorname{codim}_{\mathbb{C}} \bigvee_{i=1}^{k} A_{i}$.

Comparing Proposition 1 with Theorem 1, we have a generalization of Proposition 1.1 from [2], where only the case of $g$ being the minimal building set, i.e., the set of irreducibles, is considered.

Corollary 2. Let $\mathcal{A}$ be an essential arrangement of complex hyperplanes, $\mathcal{L}=\mathscr{L}(\mathcal{A})$ its intersection lattice, and $g$ a building set in $\mathcal{L}$ containing $\{0\}$. Then the cohomology algebra of the arrangement compactification $\overline{Y_{q}}$ is isomorphic to the algebra $D(\mathcal{L}, \mathcal{G})$ defined in Sect. 2:

$$
H^{*}\left(\overline{Y_{g}}\right) \cong D(\mathcal{L}, g) \text {. }
$$

In the rest of the section we will give several examples of the Poincare series for compactifications of hyperplane arrangement complements. This means we compute the Hilbert series of $D(\mathcal{L}, \mathscr{g})$. We restrict our computations to the compactifications with $g$ being the maximal building set $\mathscr{L} \backslash\{\hat{0}\}$, although they can be easily generalized to arbitrary $g$. 
For these examples we use the basis of $D(\mathscr{L})=D(\mathcal{L}, \mathcal{L} \backslash\{\hat{0}\})$ from Corollary 1. In the considered case the basic monomials are parametrized by certain flags in $\mathscr{L} \backslash\{\hat{0}\}$ with multiplicity assigned to their elements. The upper bounds for multiplicities allow us to write the Hilbert series of $\mathrm{D}(\mathscr{L})$ in the following form. For each sequence $r$ of natural numbers, $r=\left(0=r_{0}<r_{1}<\cdots<r_{k} \leq \mathrm{rk} \mathcal{L}\right)$ denote by $f_{\mathcal{L}}(r)$ the number of flags in $\mathcal{L}$ whose sequence of ranks equals $r$. Set $k=k(r)$ and call it the length of $r$. Then we have

$$
H(\mathrm{D}(\mathcal{L}), t)=1+\sum_{r}\left[\prod_{i=1}^{k(r)} \frac{t(1-t)^{r_{i}-r_{i-1}-1}}{1-t}\right] f_{\mathscr{L}}(r) .
$$

Here, $r$ runs over all sequences as above and we use the agreement $\frac{t(t-1)^{0}}{t-1}=1$.

In some important cases one can give more explicit descriptions of the numbers $f_{\mathscr{L}}(r)$ whence of the Hilbert series. We consider two such cases.

Generic arrangements. For arrangements from this class, the intersection lattice $\mathcal{L}$ is defined by the number $n$ of atoms and the rank $\ell$. We use both pieces of notation: $\mathcal{L}$ and $\mathcal{L}(n, \ell)$. The number of elements of $\mathcal{L}$ of rank $\ell^{\prime}<\ell$ is $\left(\begin{array}{l}n \\ \ell^{\prime}\end{array}\right)$ and for every $X \in \mathcal{L}$ of rank $\ell^{\prime}$ the lattice $\{Y \in \mathscr{L} \mid Y \geq X\}$ is isomorphic to $\mathcal{L}\left(n-\ell^{\prime}, \ell-\ell^{\prime}\right)$. This immediately implies the following formula:

$$
f_{\mathscr{L}}(r)=\prod_{i=1}^{k}\left(\begin{array}{c}
n-r_{i-1} \\
r_{i}-r_{i-1}
\end{array}\right),
$$

where $k=k(r)$ if $r_{k(r)}<\ell$ and $k=k(r)-1$ otherwise. This gives

$$
\begin{aligned}
& H(\mathrm{D}(\mathcal{L}(n, \ell)), t)= \\
& 1+\sum_{r}\left\{\left[1+\frac{t(1-t)^{\ell-r_{k}-1}}{1-t}\right] \prod_{i=1}^{k(r)} \frac{t(1-t)^{r_{i}-r_{i-1}-1}}{1-t}\left(\begin{array}{c}
n-r_{i-1} \\
r_{i}-r_{i-1}
\end{array}\right)\right\},
\end{aligned}
$$

where the summation now is over all $r$ with the extra condition $r_{k(r)}<\ell$ and we again use the agreement $\frac{t(t-1)^{0}}{t-1}=1$.

Braid arrangements. For the rank $n-1$ complex braid arrangement (compare Example 1) the intersection lattice is given by the partition lattice $\Pi_{n}$ of set partitions of $[n]:=\{1, \ldots, n\}$ ordered by reverse refinement. Observe that the rank of a partition $\pi$ coincides with $n-|\pi|$ where $|\pi|$ is the number of blocks of the partition. Thus the number of elements of $\Pi_{n}$ of rank $\ell$ is $p_{n-\ell}(n)$ that is the number of partitions of $[n]$ in $n-\ell$ blocks. For every $X \in \Pi_{n}$ of rank $\ell$ the lattice $\left\{Y \in \Pi_{n} \mid Y \geq X\right\}$ is isomorphic to $\Pi_{n-\ell}$. This immediately implies the following formulas:

$$
f_{\Pi_{n}}(r)=\prod_{i=1}^{k(r)} p_{n-r_{i}}\left(n-r_{i-1}\right)
$$


and

$$
H\left(\mathrm{D}\left(\Pi_{n}\right), t\right)=1+\sum_{r}\left[\prod_{i=1}^{k(r)} \frac{t(1-t)^{r_{i}-r_{i-1}-1}}{1-t} p_{n-r_{i}}\left(n-r_{i-1}\right)\right]
$$

where the summation is over all $r$.

\section{The toric variety $X_{\Sigma(\mathscr{L}, q)}$}

In this section we present another geometric interpretation of the algebra $D(\mathcal{L}, \mathcal{G})$, this time for an arbitrary atomic lattice $\mathcal{L}$. For a given building set $\mathcal{g}$ in $\mathcal{L}$ we construct a toric variety $X_{\Sigma(\mathcal{L}, g)}$ and show that its Chow ring is isomorphic to the algebra $D(\mathcal{L}, \mathscr{g})$.

Given a finite lattice $\mathcal{L}$ with set of atoms $\mathfrak{A}(\mathcal{L})=\left\{A_{1}, \ldots, A_{n}\right\}$, we will frequently use the following notation. For $X \in \mathcal{L}$, denote the set of atoms below $X$ by $\lfloor X\rfloor:=\{A \in \mathfrak{A}(\mathcal{L}) \mid X \geq A\}$. Define characteristic vectors $v_{X}$ in $\mathbb{R}^{n}$ for $X \in \mathcal{L}$ with coordinates

$$
\left(v_{X}\right)_{i}:=\left\{\begin{array}{l}
1 \text { if } A_{i} \in\lfloor X\rfloor, \\
0 \text { otherwise, }
\end{array} \quad \text { for } i=1, \ldots, n .\right.
$$

We will consider cones spanned by these characteristic vectors. We therefore agree to denote by $V(\delta)$ the cone spanned by the vectors $v_{X}$ for $X \in \delta$, $\delta \subseteq \mathcal{L}$.

Let $\mathcal{L}$ be a finite atomic lattice and $\mathcal{G}$ a building set in $\mathcal{L}$. We define a rational, polyhedral fan $\Sigma(\mathcal{L}, \mathcal{G})$ in $\mathbb{R}^{n}$ by taking cones $V(\mathscr{S})$ for any nested set $\delta$ in $\mathcal{L}$,

$$
\Sigma(\mathcal{L}, \mathcal{g}):=\{V(\delta) \mid \delta \in \mathcal{N}(\mathscr{L}, \mathscr{g})\} .
$$

By definition, rays in $\Sigma(\mathcal{L}, \mathscr{g})$ are in 1-1 correspondence with elements in $\mathcal{G}$; the face poset of $\Sigma(\mathcal{L}, \mathcal{G})$ coincides with the face poset of $\mathcal{N}(\mathcal{L}, \mathcal{G})$. To specify the set of cones in $\Sigma(\mathcal{L}, \mathcal{g})$ of a fixed dimension $k$, or nested sets in $\mathcal{G}$ with $k$ elements, we often use the notation $\Sigma(\mathcal{L}, \mathcal{G})_{k}$ or $\mathcal{N}(\mathcal{L}, \mathcal{G})_{k}$, respectively.

Proposition 2. The polyhedral fan $\Sigma(\mathcal{L}, \mathcal{g})$ is unimodular.

Proof. We need to show that for any nested set $s \in \mathcal{N}(\mathcal{L}, \mathcal{Q})$ the set of generating vectors for $V(\&),\left\{v_{X} \mid X \in \delta\right\}$, can be extended to a lattice basis for $\mathbb{Z}^{n}$. To that end, fix a linear order $\prec$ on $\&$ that refines the given order on $\mathcal{L}$, and write the generating vectors $v_{X}$ as rows of a matrix $A$ following this linear order. Now transform $A_{\widetilde{X}}$ to a matrix $\widetilde{A}$, replacing each vector $v_{X}$ by the characteristic vector $v_{\widetilde{X}}$ of $\widetilde{X}$, with

$$
\tilde{X}=\bigvee_{\substack{Y \in S \\ Y \leq X}} Y
$$


For each $X$ this can be done by adding rows $v_{Z}$ to $v_{X}$ for elements

$$
Z \in \max _{\mathcal{L}}\{Y \in \mathcal{S} \mid Y \prec X, Y \text { incomparable to } X \text { in } \mathcal{L}\},
$$

the reason being that characteristic sets of atoms for incomparable elements of a nested set are disjoint [4, Prop. 2.5(1),2.8]. The matrix $\widetilde{A}$ clearly has rows with strictly increasing support, hence can be easily extended to a square matrix with determinant \pm 1 . The same extra rows will complete the rows of the original matrix $A$ to a lattice basis for $\mathbb{Z}^{n}$.

Remark 1. In Sect. 6 we will give a more constructive description of $\Sigma(\mathcal{L}, \mathcal{G})$, picturing the fan as the result of successive stellar subdivisions of faces of the $n$-dimensional cone spanned by the standard lattice basis for $\mathbb{Z}^{n}$ and subsequent removal of faces (compare Thm. 4). From this description, unimodality of the fan will follow immediately.

Let $X_{\Sigma(\mathcal{L}, \mathscr{g})}$ denote the toric variety associated with $\Sigma(\mathscr{L}, \mathscr{q})$. If there is no risk of confusion, we will abbreviate notation by using $X_{\Sigma}$ instead. $X_{\Sigma}$ is a smooth, non-complete, complex algebraic variety. Crucial for us will be its stratification by torus orbits $\mathcal{O}_{\S}$, in one-to-one correspondence with cones $V(\mathscr{S})$ in $\Sigma(\mathcal{L}, \mathcal{G})$, thus with nested sets $\&$ in $g$.

The orbit closures $\left[\mathcal{O}_{\&}\right], \delta \in \mathcal{N}(\mathcal{L}, \mathscr{g})_{n-k}$, generate the Chow groups $A_{k}\left(X_{\Sigma}\right), k=0, \ldots, n$. We describe generators for the groups of relations among the $\left[\mathcal{O}_{s}\right], \delta \in \mathcal{N}(\mathscr{L}, \mathscr{Q})_{n-k}$, in $A_{k}\left(X_{\Sigma}\right)$ for later reference. This description is due to Fulton and Sturmfels [6]. We present here a slight adaptation to our present context.

Proposition 3. ([6, 2.1]) The group of relations among generators $\left[\mathcal{O}_{8}\right]$, $s \in \mathcal{N}(\mathcal{L}, \mathcal{Q})_{n-k}$, for the $k$-th Chow group $A_{k}\left(X_{\Sigma}\right), k=0, \ldots, n$, is generated by relations of the form

$$
r(\mathcal{T}, b)=\sum_{\substack{\delta \supset \mathcal{T} \\ \delta \in \mathcal{N}(\mathcal{L}, \mathscr{g})_{n-k}}}<b, z_{\S, \mathcal{T}}>\left[\mathcal{O}_{\S}\right],
$$

where $\mathcal{T}$ runs over all nested sets with $n-k-1$ elements and $b$ over a generating set for the sublattice determined by $V(\mathcal{T})^{\perp}$ in the dual lattice $\operatorname{Hom}\left(\mathbb{Z}^{n}, \mathbb{Z}\right)$. Here, $z_{s, \mathcal{T}}$ is a lattice point in $V(\&)$ generating the (1-dimensional) lattice $\operatorname{span}\left(V(\&) \cap \mathbb{Z}^{n}\right) / \operatorname{span}\left(V(\mathcal{T}) \cap \mathbb{Z}^{n}\right)$.

Since $X_{\Sigma(\mathcal{L}, q)}$ is non-singular, the intersection product $\cdot$ makes $\mathrm{Ch}^{*}\left(X_{\Sigma}\right)$ $=\oplus_{k=0}^{n} \mathrm{Ch}^{k}\left(X_{\Sigma}\right)$ with $\mathrm{Ch}^{k}\left(X_{\Sigma}\right)=A_{n-k}\left(X_{\Sigma}\right)$ into a commutative graded ring, the Chow ring of $X_{\Sigma(\mathcal{L}, q)}$.

Theorem 3. Let $X_{\Sigma(\mathcal{L}, q)}$ be the toric variety associated with a finite atomic lattice $\mathcal{L}$ and a combinatorial building set $\mathcal{G}$ in $\mathcal{L}$ as described above. Then the assignment $x_{G} \mapsto\left[\mathcal{O}_{\{G\}}\right]$ for $G \in \mathcal{G}$, extends to an isomorphism

$$
D(\mathcal{L}, \mathcal{g}) \cong \mathrm{Ch}^{*}\left(X_{\Sigma(\mathcal{L}, \mathscr{g})}\right) \text {. }
$$


Proof. Orbit closures $\left[\mathcal{O}_{\{G\}}\right]$ in $X_{\Sigma}$ that correspond to the rays $V(\{G\})$ in $\Sigma(\mathcal{L}, \mathcal{G})$ for $G \in \mathcal{G}$, generate $\mathrm{Ch}^{*}\left(X_{\Sigma}\right)$ multiplicatively, since

$$
\left[\mathcal{O}_{s}\right]=\left[\mathcal{O}_{\left\{G_{1}\right\}}\right] \cdot \ldots \cdot\left[\mathcal{O}_{\left\{G_{k}\right\}}\right]
$$

for $\delta=\left\{G_{1}, \ldots, G_{k}\right\} \in \mathcal{N}(\mathcal{L}, \mathcal{G})$, denoting the intersection product (see $[5$, p. 100]).

Moreover, relations as in $D(\mathcal{L}, \mathcal{g})$ hold. Indeed, the intersection products of orbit closures corresponding to rays that do not span a cone in $\Sigma(\mathcal{L}, \mathcal{g})$ are 0 [5, p. 100], which is exactly the monomial relations (1) for non-nested index sets in $D(\mathcal{L}, \mathcal{g})$. Relations (14) in $\mathrm{Ch}^{1}\left(X_{\Sigma}\right)=A_{n-1}\left(X_{\Sigma}\right)$ as described above coincide with the linear relations $(2)$ in $D(\mathcal{L}, \mathcal{E})$

$$
r\left(\emptyset, v_{A}\right)=\sum_{G \in \mathcal{G}}<v_{A}, v_{G}>\left[\mathcal{O}_{\{G\}}\right]=\sum_{G \geq A}\left[\mathcal{O}_{\{G\}}\right]
$$

the $v_{A}$, for $A \in \mathfrak{A}(\mathcal{L})$, forming a basis for the lattice orthogonal to $V(\emptyset)=0$ in $\mathbb{Z}^{n}$.

Thus, sending $x_{G}$ to $\left[\mathcal{O}_{\{G\}}\right]$ for $G \in \mathcal{G}$, we have a surjective ring homomorphism from $D(\mathcal{L}, \mathcal{g})$ to the Chow ring of $X_{\Sigma}$. It remains to show that the relations (14) in $\mathrm{Ch}^{*}\left(X_{\Sigma}\right)$ follow from relations (15) in $\mathrm{Ch}^{1}\left(X_{\Sigma}\right)$, and from monomials over non-nested index sets being zero.

Let us fix some notation. For $\mathcal{T} \in \mathcal{N}(\mathcal{L}, \mathscr{g})$ and $X \in \mathcal{T}$ define

$$
\Delta_{\mathcal{T}}(X):=\lfloor X\rfloor \backslash \bigcup_{\substack{Y<X \\ Y \in \mathcal{T}}}\lfloor Y\rfloor,
$$

the set of atoms that are below $X$, but not below the join of all $Y$ in $\mathcal{T}$ that are smaller than $X$. Observe that $\Delta_{\mathcal{T}}(X) \neq \emptyset$ for any $X \in \mathcal{T}$, since $\mathcal{T}$ is nested, and

$$
\lfloor\bigvee \mathcal{T}\rfloor=\bigcup_{X \in \mathcal{T}} \Delta_{\mathcal{T}}(X)
$$

For $\mathcal{T} \in \mathcal{N}(\mathcal{L}, \mathcal{g})_{k-1}, k \geq 2$, the sublattice determined by $V(\mathcal{T})^{\perp}$ in the dual lattice is generated by vectors in $\mathcal{C}_{1} \cup \mathcal{C}_{2}$, where

$$
\begin{aligned}
& \mathcal{C}_{1}=\left\{v_{A_{i}}-v_{A_{j}} \mid A_{i}, A_{j} \in \Delta_{\mathcal{T}}(X) \text { for some } X \in \mathcal{T}\right\}, \\
& \mathcal{C}_{2}=\left\{v_{A} \mid A \in \mathfrak{A}(\mathcal{L}) \backslash\lfloor\bigvee \mathcal{T}\rfloor\right\} .
\end{aligned}
$$

Observe that $\mathcal{C}_{1} \cup \mathcal{C}_{2}$ contains $\sum_{X \in \mathcal{T}}\left(\left|\Delta_{\mathcal{T}}(X)\right|-1\right)+|\mathfrak{A}(\mathcal{L}) \backslash\lfloor\bigvee \mathcal{T}\rfloor|=$ $|\mathfrak{A}(\mathcal{L})|-|\mathcal{T}|=\operatorname{codim} V(\mathcal{T})$ linear independent vectors, thus a basis for the sublattice determined by $V(\mathcal{T})^{\perp}$. 
For $\mathcal{T} \in \mathcal{N}(\mathcal{L}, \mathscr{Q})_{k-1}, k \geq 2$, and $v_{A_{i}}-v_{A_{j}} \in \mathcal{C}_{1}$, the relation (14) reads as

$$
\begin{aligned}
& r\left(\mathcal{T}, v_{A_{i}}-v_{A_{j}}\right) \\
& =\sum_{\substack{\delta \supset \mathcal{T} \\
\delta \in \mathcal{N}(\mathscr{L}, \mathcal{f})_{k}}}<v_{A_{i}}-v_{A_{j}}, z_{\delta, \mathcal{T}}>\left[\mathcal{O}_{\delta}\right] \\
& =\sum_{\substack{Y \in \mathcal{G} \backslash \mathcal{T} \\
\mathcal{T} \cup\{Y\} \in \mathcal{N}(\mathcal{L}, \mathcal{G})}}<v_{A_{i}}-v_{A_{j}}, v_{Y}>\left[\mathcal{O}_{\mathcal{T} \cup\{Y\}}\right] \\
& =\left[\mathcal{O}_{\mathcal{T}}\right] \cdot\left(\sum_{\substack{Y \in \mathcal{G} \mid \mathcal{T}, Y \geq A_{i} \\
\mathcal{T} \cup\{Y\} \in \mathcal{N}(\mathcal{L}, \mathcal{F})}}\left[\mathcal{O}_{\{Y\}}\right]-\sum_{\substack{Y \in \mathcal{S} \backslash \mathcal{T}, Y \geq A_{j} \\
\mathcal{T} \cup\{Y\} \in \mathcal{N}(\mathcal{L}, \mathcal{G})}}\left[\mathcal{O}_{\{Y\}}\right]\right) .
\end{aligned}
$$

Monomials over non-nested index sets being zero, we may drop the condition $\mathcal{T} \cup\{Y\} \in \mathcal{N}(\mathcal{L}, \mathcal{G})$ in both sums. Moreover, if $Y \in \mathcal{T}, Y$ either is larger than both $A_{i}$ and $A_{j}$, or not larger than either of them. Thus, both sums in $r\left(\mathcal{T}, v_{A_{i}}-v_{A_{j}}\right)$ are relations of type (15), hence $r(\mathcal{T}, c), c \in \mathcal{C}_{1}$, is a consequence of relations of type (1) and (2) holding in $\mathrm{Ch}^{*}\left(X_{\Sigma}\right)$, as claimed.

For $v_{A} \in \mathcal{C}_{2}$, the reasoning is similar, but easier. Indeed

$$
\begin{aligned}
r\left(\mathcal{T}, v_{A}\right) & =\sum_{\substack{\delta \supset \mathcal{T} \\
s \in \mathcal{N}(\mathscr{L}, \mathscr{q})_{k}}}<v_{A}, z_{s, \mathcal{T}}>\left[\mathcal{O}_{s}\right] \\
& =\sum_{\substack{Y \in \mathcal{G} \backslash \mathcal{T} \\
\mathcal{T} \cup\{Y\} \in \mathcal{N}(\mathcal{L}, \mathscr{q})}}<v_{A}, v_{Y}>\left[\mathcal{O}_{\mathcal{T} \cup\{Y\}}\right] \\
& =\left[\mathcal{O}_{\mathcal{T}}\right] \cdot \sum_{Y \geq A}\left[\mathcal{O}_{\{Y\}}\right],
\end{aligned}
$$

since no $Y \in \mathcal{T}$ can be larger than $A$, and again, by monomials over nonnested sets being zero, the condition $\mathcal{T} \cup\{Y\} \in \mathcal{N}(\mathcal{L}, \mathcal{G})$ can be dropped. This completes our proof.

\section{A geometric description of $X_{\Sigma(\mathcal{L}, q)}$}

The goal of this section is to give a geometric description of the variety $X_{\Sigma(\mathcal{L}, g)}$. For an arbitrary atomic lattice $\mathcal{L}$, we describe the toric variety $X_{\Sigma(\mathcal{L}, \mathcal{g})}$ as the result of a sequence of blowups of closed torus orbits and subsequent removal of a number of open orbits. We start with a more constructive description of the fan $\Sigma(\mathcal{L}, \mathscr{Q})$ as the result of a sequence of stellar subdivisions and subsequent removal of a number of open cones. 
We allow the same setting as for the definition of $\Sigma(\mathcal{L}, \mathcal{F})$ in (13). Let $\mathcal{L}$ be a finite atomic lattice with set of atoms $\mathfrak{A}(\mathcal{L})=\left\{A_{1}, \ldots, A_{n}\right\}$ and $\mathscr{q}$ a building set in $\mathcal{L}$.

\section{Construction of $\Theta(\mathcal{L}, q)$.}

(0) Start with the fan $\Theta_{0}$ given by the $n$-dimensional cone spanned by the coordinate vectors in $\mathbb{R}^{n}$ together with all its faces.

(1) Choose a linear order $\succ$ on $g$ that is non-increasing with respect to the original partial order on $\mathcal{L}$, i.e., $G \leq G^{\prime}$ implies $G^{\prime} \succeq G$. Write $\mathscr{Q}=\left\{G_{1} \succ\right.$ $\left.G_{2} \succ \cdots \succ G_{t}\right\}$. Construct a fan $\widetilde{\Theta}(\mathcal{L}, \mathscr{g})$ by successive barycentric stellar subdivisions in faces $V\left(\left\lfloor G_{i}\right\rfloor\right)$ of $\Theta_{0}$ for $i=1, \ldots, t$, introducing in each step a new ray generated by the characteristic vector $v_{G_{i}}, i=1, \ldots, t$.

(2) Remove from $\widetilde{\Theta}(\mathscr{L}, \mathcal{G})$ all (open) cones $V(\mathcal{T})$ with index sets of generating vectors $\mathcal{T}$ that are not nested in $\mathcal{G}$ and denote the resulting fan by $\Theta(\mathcal{L}, \mathcal{G})$.

Theorem 4. The fan $\Theta(\mathcal{L}, \mathscr{Q})$ constructed above coincides with the fan $\Sigma(\mathcal{L}, \mathscr{g})$ defined in Sect. 5.

Proof. By construction the fans share the same generating vectors. In fact, due to the removal of cones in step (2) of the construction above, it is enough to show that for any nested set $\delta \in \mathcal{N}(\mathcal{L}, \mathcal{g})$ there exists a cone in $\widetilde{\Theta}(\mathcal{L}, \mathcal{G})$ containing $V(\delta)$ as a face. Due to the recursive construction of $\widetilde{\Theta}(\mathcal{L}, \mathcal{g})$ this statement reduces to the following claim.

Claim. Let $\delta=\left\{X_{1}, \ldots, X_{k}\right\}$ be nested in $\mathcal{L}$ with respect to $g$, and assume that the indexing is compatible with the linear order $\succ$ on $g$, i.e., $X_{1} \succ \ldots \succ X_{k}$. For notational convenience, extend the set by $X_{k+1}:=\hat{0}$. Then any stellar subdivision in $V(\lfloor G\rfloor), G \in g$, during the construction of $\widetilde{\Theta}(\mathcal{L}, g)$, for $G \succ X_{i}, G \nsucceq X_{i-1}, i=1, \ldots, k+1$, retains a cone $W_{G}$ with

$$
V\left(\left\{X_{1}, \ldots, X_{i-1}\right\} \cup\left\lfloor X_{i}\right\rfloor \cup \ldots \cup\left\lfloor X_{k}\right\rfloor\right)
$$

among its faces and for $G=X_{i}, i=1, \ldots, k$, creates a cone $W_{X_{i}}$ with

$$
V\left(\left\{X_{1}, \ldots, X_{i}\right\} \cup\left\lfloor X_{i+1}\right\rfloor \cup \ldots \cup\left\lfloor X_{k}\right\rfloor\right)
$$

among its faces.

Proof of the claim. Assume first that $G \succ X_{i}, G \nsucceq X_{i-1}$, for some $i \in$ $\{1, \ldots, k+1\}$ (the second condition being empty for $k=1$ ), and assume that the previous subdivision step in $V\left(\left\lfloor G^{\prime}\right\rfloor\right), G^{\prime} \in \mathcal{G}$, has created, resp. retained a cone $W_{G^{\prime}}$ with $V\left(\left\{X_{1}, \ldots, X_{i-1}\right\} \cup\left\lfloor X_{i}\right\rfloor \cup \ldots \cup\left\lfloor X_{k}\right\rfloor\right)$ among its faces.

If $W\left(G^{\prime}\right)$ does not contain $V(\lfloor G\rfloor)$, it will not be altered by stellar subdivision in $V(\lfloor G\rfloor)$. Any cone that is to be altered when subdividing $V(\lfloor G\rfloor)$ 
needs to be contained in star $V(\lfloor G\rfloor)$, hence among its faces needs to contain $V(\lfloor G\rfloor)$.

If $W\left(G^{\prime}\right)$ does contain $V(\lfloor G\rfloor)$ among its faces, choose

$$
g \in\lfloor G\rfloor \backslash \bigcup_{j=i}^{k}\left\lfloor X_{j}\right\rfloor .
$$

If the set was empty, we would have $\lfloor G\rfloor \subseteq \bigcup_{j \geq i}\left\lfloor X_{j}\right\rfloor$, in particular,

$$
G \leq \bigvee_{j \geq i} X_{j} \leq \bigvee_{\max \delta_{\geq X_{i}}} X_{j}
$$

The join on the right hand side is taken over all $X_{j}$ that are maximal among $X_{1}, X_{2}, \ldots, X_{i}$ with respect to the partial order in $\mathcal{L}$. Since these elements are pairwise incomparable and nested in $\mathscr{L}$ they are the factors of their join. This implies that $G \leq X_{j^{*}}$ for some $j^{*} \geq i$ [4, Prop. 2.5(i)] contradicting the fact that $G \succ X_{j^{*}}$.

Hence we can choose $g$ as described in (16) and, when subdividing $V(\lfloor G\rfloor)$, we replace $W_{G^{\prime}}$ by $W_{G}$ by substituting the new ray $\left\langle v_{G}\right\rangle$ for the ray $\left\langle v_{g}\right\rangle$ in $W_{G^{\prime}}$. Observe that $V\left(\left\{X_{1}, \ldots, X_{i-1}\right\} \cup\left\lfloor X_{i}\right\rfloor \cup \ldots \cup\left\lfloor X_{k}\right\rfloor\right)$ remains as a face in the newly created cone $W_{G}$.

Assume now that $G=X_{i}$ and again denote the cone emerging from the previous subdivision step by $W_{G^{\prime}}$, assuming that it contains $V\left(\left\{X_{1}, \ldots\right.\right.$, $\left.\left.X_{i-1}\right\} \cup\left\lfloor X_{i}\right\rfloor \cup \ldots \cup\left\lfloor X_{k}\right\rfloor\right)$ among its faces. When subdividing $V\left(\left\lfloor X_{i}\right\rfloor\right)$ now replace $W_{G^{\prime}}$ by $W_{X_{i}}$ by substituting the new ray $\left\langle v_{X_{i}}\right\rangle$ for the generating ray associated with some

$$
x_{i} \in\left\lfloor X_{i}\right\rfloor \backslash \bigcup_{j \geq i+1}\left\lfloor X_{j}\right\rfloor=\left\lfloor X_{i}\right\rfloor \backslash \bigcup_{\substack{j \geq i+1 \\ X_{j}<X_{i}}}\left\lfloor X_{j}\right\rfloor=\Delta_{s}\left(X_{i}\right),
$$

where the right hand side is non-empty as we observed before (see proof of Thm. 3).

Note that $V\left(\left\{X_{1}, \ldots, X_{i}\right\} \cup\left\lfloor X_{i+1}\right\rfloor \cup \ldots \cup\left\lfloor X_{k}\right\rfloor\right)$ is a face of the newly created cone $W_{X_{i}}$. This completes the proof of our claim.

Corollary 3. The toric variety $X_{\Sigma(\mathcal{L}, q)}$ can be constructed as follows. Start from the toric variety associated with the standard $n$-dimensional cone spanned by the standard lattice basis in $\mathbb{Z}^{n}$, i.e., from $\mathbb{C}^{n}$ stratified by torus orbits. Perform a sequence of blowups in orbit closures associated with faces $V(\lfloor G\rfloor)$ of the standard cone for $G \in G$ in some linear, non-increasing order. Remove from the resulting variety all open torus orbits that correspond to cones in $\widetilde{\Theta}(\mathcal{L}, \mathcal{G})$ indexed with non-nested subsets of $\mathcal{L}$.

It follows immediately from this description that the toric variety $X_{\Sigma(\mathcal{L}, q)}$ is smooth. 


\section{Examples}

We discuss a number of examples to illustrate the central notions of this article.

\section{Partition lattices}

Let $\Pi_{n}$ denote the lattice of set partitions of $[n]$ ordered by reversed refinement. As we mentioned above, the partition lattice $\Pi_{n}$ occurs as the intersection lattice of the braid arrangement $\mathcal{A}_{n-1}$ (compare Example 1).

For $n=3$, the only building set is the maximal one, i.e., $g=\Pi_{3} \backslash\{\hat{0}\}$. Denoting elements as in the Hasse diagram depicted on the right, the nested set complex $\mathcal{N}\left(\Pi_{3}, g\right)$ contains the following simplices:

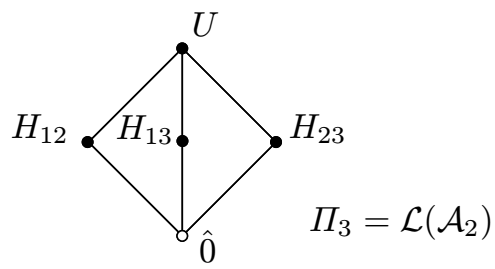

$$
\mathcal{N}\left(\Pi_{3}, \mathcal{g}\right)=\left\{H_{12}, H_{13}, H_{23}, U, H_{12} U, H_{13} U, H_{23} U\right\} .
$$

The algebra $D\left(\Pi_{3}, g\right)$ thus is the following:

$$
\begin{aligned}
D\left(\Pi_{3}, g\right)=\mathbb{Z}\left[x_{H_{12}}, x_{H_{13}}, x_{H_{23}}, x_{U}\right] & \\
& \left(\begin{array}{ll}
x_{H_{12}} x_{H_{13}}, x_{H_{12}} x_{H_{23}}, x_{H_{13}} x_{H_{23}} \\
x_{H_{12}}+x_{U}, x_{H_{13}}+x_{U}, x_{H_{23}}+x_{U}
\end{array}\right) .
\end{aligned}
$$

We find that $D\left(\Pi_{3}, g\right) \cong \mathbb{Z}\left[x_{U}\right] /\left\langle x_{U}^{2}\right\rangle$, which illustrates Corollary 2 . The compactification $\bar{Y}_{\Pi_{3} \backslash\{\hat{0}\}}$ of the complement of the projectivized braid arrangement $\mathbb{P}_{\mathcal{A}_{2}}$ (a three times punctured $\mathbb{C P}^{1}$ ) is the complex projective line.

To visualize the fan $\Sigma\left(\Pi_{3}, g\right)$ we choose to depict its intersection with a hyperplane orthogonal to the diagonal ray in the positive octant of $\mathbb{R}^{3}$. To shorten notation, we denote rays by building set elements.

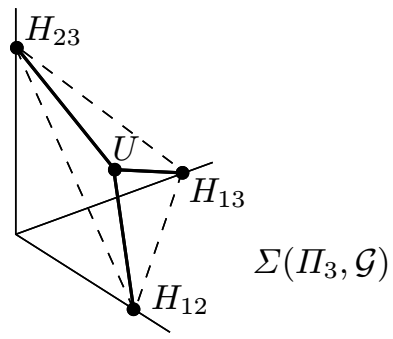

The toric variety $X_{\Sigma\left(\Pi_{3}, q\right)}$ is the blowup of $\mathbb{C}^{3}$ in 0 with open torus orbits corresponding to cones $V\left(H_{12}, H_{13}\right), V\left(H_{12}, H_{23}\right), V\left(H_{13}, H_{23}\right)$ and $V\left(H_{12}, H_{13}, U\right), V\left(H_{12}, H_{23}, U\right), V\left(H_{13}, H_{23}, U\right)$ removed. What we remove here, in fact, are the proper transforms of the three coordinate axes of $\mathbb{C}^{3}$ after blowup in 0.

For $n=4$, we have several choices when fixing a building set. The partitions with only one non-trivial block of size $\geq 2$ form the minimal building set $\mathcal{F}$. To obtain the others we add any number of 2-block partitions in $\Pi_{4}$. 


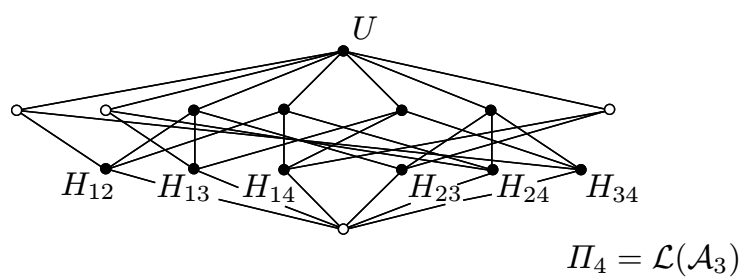

The nested set complex $\mathcal{N}\left(\Pi_{4}, \mathcal{F}\right)$ is a 2-dimensional complex on 11 vertices. It is a cone with apex $U$, the simplices in its base $\mathcal{N}\left(\Pi_{4}, \mathcal{F}\right)_{0}$ being the ordered subsets in $\mathcal{F} \backslash\{U\}$ together with the pairs $\mathrm{H}_{12} \mathrm{H}_{34}, \mathrm{H}_{13} \mathrm{H}_{24}$, $H_{14} H_{23}$. We depict below the 1-dimensional base $\mathcal{N}\left(\Pi_{4}, \mathcal{F}\right)_{0}$. To simplify notation we label vertices with the non-trivial blocks of the corresponding partitions. The non-ordered nested pairs are indicated by dotted lines.

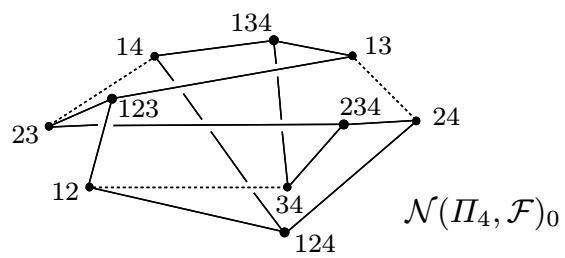

Choosing instead of $\mathcal{F}$ the maximal building sets $g$ in $\Pi_{4}$, i.e., including the 2-block partitions into the building set, results in a subdivision of these edges by additional vertices $H_{12 \mid 34}, H_{13 \mid 24}$ and $H_{14 \mid 23}$ corresponding to the newly added building set elements.

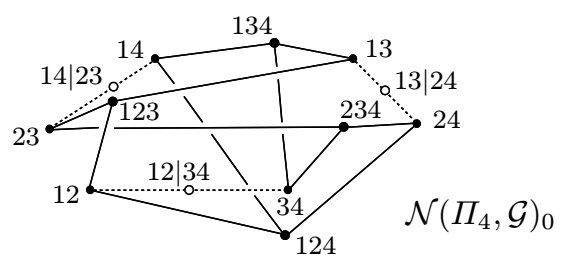

Simplifying the presentation of the algebra $D\left(\Pi_{4}, \mathcal{F}\right)$ given in Definition 3 yields

$$
\begin{aligned}
D\left(\Pi_{4}, \mathcal{F}\right) \cong \mathbb{Z}\left[x_{123}, x_{124}, x_{134}, x_{234}, x_{U}\right] / \\
\left.\qquad \begin{array}{ll}
x_{i j k} x_{U} & \text { for all } 1 \leq i<j<k \leq 4 \\
x_{i j k} x_{i^{\prime} j^{\prime} k^{\prime}} & \text { for all } i j k \neq i^{\prime} j^{\prime} k^{\prime} \\
x_{i j k}^{2}+x_{U}^{2} & \text { for all } 1 \leq i<j<k \leq 4
\end{array}\right),
\end{aligned}
$$

where we index generators corresponding to rank 2 lattice elements by the non-trivial blocks of the respective partitions. The linear basis described in Corollary 1 is given by the monomials $x_{123}, x_{124}, x_{134}, x_{234}, x_{U}$, and $x_{U}^{2}$.

For completeness, we state the description of $D\left(\Pi_{n}, \mathcal{F}\right)$ for general $n$, where $\mathcal{F}$ again denotes the minimal building set, i.e., the set of 1-block 
partitions in $\Pi_{n}$. Having in mind that $D\left(\Pi_{n}, \mathcal{F}\right)$ is isomorphic to the cohomology of the Deligne-Knudson-Mumford compactification $\bar{M}_{0, n+1}$ of the moduli space of $n+1$-punctured complex projective lines (compare Example 1), the following presentation should be compared with presentations for $H^{*}\left(\bar{M}_{0, n+1}\right)$ given earlier by Keel [8].

We index generators for $D\left(\Pi_{n}, \mathcal{F}\right)$ with subsets of $[n]$ of cardinality larger than two representing the non-trivial blocks in the respective partitions and obtain:

$$
\begin{array}{ll}
D\left(\Pi_{n}, \mathcal{F}\right) \cong \mathbb{Z}\left[\left\{x_{S}\right\}_{S \subseteq[n],|S| \geq 2}\right] / & \text { for } S \cap T \neq \emptyset, \\
& \left.\begin{array}{ll}
x_{S} x_{T} & \text { and } S \nsubseteq T, T \nsubseteq S, \\
\sum_{\{i, j\} \subseteq S} x_{S} & \text { for } 1 \leq i<j \leq n
\end{array}\right) .
\end{array}
$$

\section{A non-geometric lattice}

Consider the lattice $\mathcal{L}$ depicted by its Hasse diagram on the right. We obtain the following building sets:

$$
\begin{aligned}
& g_{1}=\left\{A_{1}, A_{2}, A_{3}, U\right\}, \\
& g_{2}=\left\{A_{1}, A_{2}, A_{3}, Y_{1}, U\right\}, \\
& g_{3}=\left\{A_{1}, A_{2}, A_{3}, Y_{1}, Y_{2}, U\right\},
\end{aligned}
$$

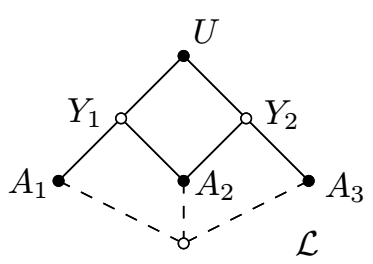

the only other choice being to replace $Y_{1}$ by $Y_{2}$ in $g_{2}$.

For a description of the nested set complexes we refer to the corresponding fans $\Sigma\left(\mathscr{L}, g_{i}\right), i=1,2,3$, shown below. The standard presentations for $D\left(\mathcal{L}, g_{i}\right), i=1,2,3$, according to Definition 3 simplify so as to reveal the Hilbert functions of the algebras to be

$$
H\left(D\left(\mathcal{L}, g_{i}\right), t\right)=1+i t \quad \text { for } i=1,2,3,
$$

with basis in degree 1 being the generators associated to building set elements other than atoms.

We depict the fans $\Sigma\left(\mathcal{L}, g_{i}\right), i=1,2,3$, again by drawing their intersections with a hyperplane orthogonal to the diagonal ray in the positive octant of $\mathbb{R}^{3}$.
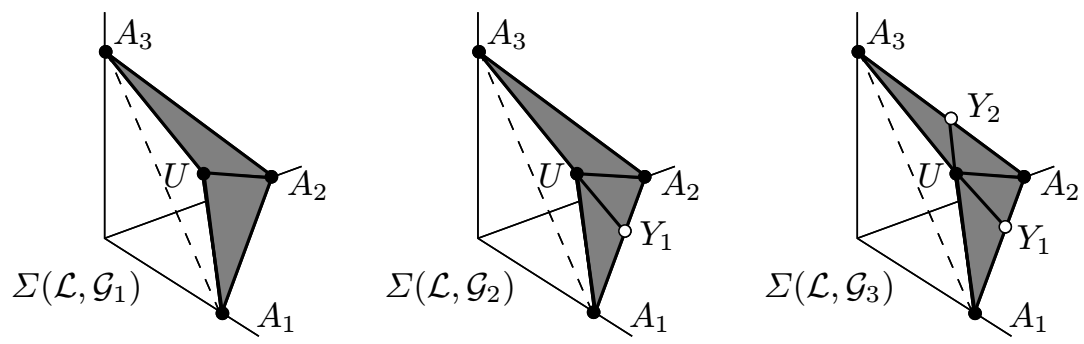
The toric variety $X_{\Sigma\left(\mathscr{L}, q_{1}\right)}$ is the result of blowing up $\mathbb{C}^{3}$ at the origin, and henceforth removing the open torus orbits corresponding to one original 2-dimensional cone and the unique 3-dimensional cone containing it.

The toric varieties $X_{\Sigma\left(\mathcal{L}, q_{2}\right)}$ and $X_{\Sigma\left(\mathcal{L}, q_{3}\right)}$ differ from $X_{\Sigma\left(\mathcal{L}, q_{1}\right)}$ by blowups in one, resp. two of the original 1-dimensional torus orbits before removing open orbits as above.

\section{References}

1. De Concini, C., Procesi, C.: Wonderful models of subspace arrangements. Sel. Math. 1, 459-494 (1995)

2. De Concini, C., Procesi, C.: Hyperplane arrangements and holonomy equations. Sel. Math. 1, 495-535 (1995)

3. Eisenbud, D.: Commutative Algebra with a View Toward Algebraic Geometry. New York: Springer 1994

4. Feichtner, E.M., Kozlov, D.N.: Incidence combinatorics of resolutions. math. CO/0305154. To appear in Sel. Math.

5. Fulton, W.: Introduction to Toric Varieties. Annals of Mathematics Studies 131. Princeton University Press 1993

6. Fulton, W., Sturmfels, B.: Intersection theory on toric varieties. Topology 36, 335-353 (1997)

7. Gaiffi, G.: Blow-ups and cohomology bases for De Concini-Procesi models of subspace arrangements. Sel. Math. 3, 315-333 (1997)

8. Keel, S.: Intersection theory of moduli space of stable $n$-pointed curves of genus zero. Trans. Am. Math. Soc. 330, 545-574 (1992)

9. Yuzvinsky, S.: Cohomology basis for the De Concini-Procesi models of hyperplane arrangements and sums over trees. Invent. Math. 127, 319-335 (1997) 